\title{
Relación entre inteligencia emocional y rendimiento académico en estudiantes universitarios peruanos
}

\author{
Relationship between emotional \\ intelligence and academic performance \\ in peruvian university students
}

\author{
Relação entre inteligência emocional \\ e desempenho acadêmico em estudantes \\ universitários peruanos
}

\author{
Dany Isela Idrogo Zamora ${ }^{1}$ \&, \\ https://orcid.org/0000-0002-5546-5943 \\ José Ander Asenjo-Alarcón \\ https://orcid.org/0000-0002-1059-4258
}

https://doi.org/10.53287/ryfs1548js42x

Fecha de ingreso: 23 de enero de 2021

Fecha de aprobación: 26 de abril de 2021

Conflictos de interés: Los autores declaran no tener conflictos de intereses

RESUMEN

La inteligencia emocional es el conjunto de capacidades que permiten a un estudiante el manejo adecuado de sus emociones y de las relaciones interpersonales, cuando es sometido a situaciones estresantes, como el ámbito educativo; en el que tienen que lidiar con conflictos internos y externos para un buen desempeño acadé-

1 Centro de Salud Chancay Baños, Cajamarca, Perú. Licenciada en Enfermería. Enfermera en el Primer Nivel de Atención, egresada de la Universidad Nacional Autónoma de Chota. Correo electrónico.: diidrogoz@gmail.com

- Facultad de Ciencias de la Salud, Universidad Nacional Autónoma de Chota, Cajamarca, Perú. Licenciado en Enfermería, Doctor en Ciencias de la Salud. Docente universitario desde el año 2013, responsable de la Unidad de Investigación de la Facultad de Ciencias de la Salud de la Universidad Nacional Autónoma de Chota del 2017 al 2019, asesor y jurado evaluador de más de 20 tesis. Correo electrónico.: ander1213@hotmail.com 
mico. La investigación tuvo como objetivo relacionar la inteligencia emocional y el rendimiento académico en los estudiantes de la Universidad Nacional Autónoma de Chota, Perú. El estudio fue descriptivo correlacional, no experimental transversal, y se trabajó con 325 estudiantes universitarios que firmaron el consentimiento informado, luego de conocer el propósito de la investigación; la inteligencia emocional se determinó con el Test de Inteligencia Emocional de BarOn ICE y el rendimiento académico con una ficha de recolección de datos. Los resultados muestran mayor proporción de estudiantes universitarios con inteligencia emocional alta $(51,1 \%)$ y rendimiento académico promedio de 12,59 $\pm 1,17$ de puntaje ponderado, resultados similares a los reportados por diversos estudios nacionales e internacionales. Se concluye que existe una relación estadísticamente significativa entre la inteligencia emocional, la dimensión adaptabilidad y el rendimiento académico de los estudiantes universitarios ( $p=0,043$ y $p=0,021)$.

\section{PALABRAS CLAVE}

Educación Basada en Competencias; Emociones; Estudiantes; Inteligencia Emocional; Rendimiento Académico (Fuente: DeCS BIREME).

\section{ABSTRACT}

Emotional intelligence is the set of capacities that allow a student to properly manage their emotions and interpersonal relationships, when subjected to stressful situations, such as the educational environment; in which they have to deal with internal and external conflicts for good academic performance. The research aimed to relate emotional intelligence and academic performance in students of the National Autonomous University of Chota, Peru. The study was descriptive correlational, not cross-sectional experimental, and it worked with 325 university students who signed the informed consent, after knowing the purpose of the research; emotional intelligence was determined with the BarOn ICE Emotional Intelligence Test and academic performance with a data collection sheet. The results show a higher proportion of university students with high emotional intelligence (51.1\%) and average academic performance of $12.59 \pm 1.17$ weighted score, results similar to those reported by various national and international studies. It is concluded that there is a statistically significant relationship between emotional intelligence, the adaptability dimension and the academic performance of university students $(p=0.043$ and $p=0.021)$.

\section{KEY WORDS}

Competency-Based Education; Emotions; Students; Emotional Intelligence; Academic Performance (Source: MeSH).

\section{RESUMO}

Inteligência emocional é o conjunto de habilidades que permitem ao aluno gerenciar adequadamente suas emoções e relações interpessoais, quando submetido a situações estressantes, como o ambiente educacional; em que têm que lidar com conflitos internos e externos para um bom desempenho acadêmico. O objetivo da pesquisa foi relacionar inteligência emocional e desempenho acadêmico em alunos da Universidade Nacional Autônoma de Chota, Peru. O estudo foi descritivo correlacional, não transversal experimental, e trabalhou com 325 universitários que assinaram o Termo de Consentimento Livre e Esclarecido, após conhecer o objetivo da pesquisa; a inteligência emocional foi determinada com o BarOn ICE Emotional Intelligence Test e o desempenho acadêmico com uma planilha de coleta de da- 
dos. Os resultados mostram maior proporção de universitários com alta inteligência emocional $(51,1 \%)$ e desempenho acadêmico médio de $12,59 \pm 1,17$ pontuação ponderada, resultados semelhantes aos relatados por diversos estudos nacionais e internacionais. Conclui-se que existe relação estatisticamente significativa entre a inteligência emocional, a dimensão adaptabilidade e o desempenho acadêmico dos universitários $(p=0,043$ e $p=0,021)$.

\section{PALAVRAS CHAVE}

Educação Baseada em Competências; Emoções; Estudantes; Inteligência Emocional; Desempenho Acadêmico (Fonte: DeCS BIREME).

\section{Introducción}

La inteligencia emocional (IE) ha estado por años desvinculada del ámbito educativo, es un factor importante que interfiere en el rendimiento académico y en las distintas facetas de los universitarios, sin embargo, su abordaje como parte de la formación profesional es ínfimo (Tariq et ál., 2020). La IE es la capacidad que debe tener un estudiante para manejar eficazmente sus emociones en el proceso de adaptación a un nuevo contexto, teniendo en cuenta dominios inherentes a su persona (intrapersonales), de relación con sus semejantes (interpersonales), aplicación de estrategias efectivas que minimicen el impacto (adaptabilidad) y respuestas adecuadas a la presión (manejo del estrés), especialmente positivas (estado de ánimo) (Ain et ál., 2021); con la finalidad de tomar decisiones asertivas, mantener vínculos personales saludables, y gestionar sus emociones favorablemente.

Asimismo, le permitirá ser tolerante frente el estrés, controlar sus impulsos, generarle bienestar y optimismo, aun cuando presente dificultades durante la trayectoria académica. Sin embargo; la IE de los estudiantes universitarios todavía no es abordada con la debida importancia, según reportes de estudios realizados en distintas ciudades del Perú, que encontraron un nivel regular y bajo de IE en los estudiantes, con $86,8 \%$ y $54 \%$, lo que evidencia un manejo inexacto de las emociones
(Palomino, 2018; Ocoruro, 2021). Por otro lado, Loyola (2018) y Nuñez (2018), presentan resultados de IE promedio en los estudiantes, con el $72 \%$ y $37,9 \%$, respectivamente, que equivale también a un nivel regular. Cifras que revelan la aplicación parcial, incompleta o no contextualizadas de estrategias para el manejo adecuado de las emociones en el ámbito educativo, lo que representa el vacío que debe saldarse mediante contribuciones científicas.

Una IE óptima en los estudiantes coadyuva a relaciones interpersonales eficaces, facilita la adaptación, mejora la capacidad de resiliencia, promueve el trabajo en equipo, las habilidades de comunicación y de aprendizaje (Barragán et ál., 2021). Por ello, la construcción de habilidades emocionales constituye el recurso que pronostica el desenvolvimiento eficiente de los estudiantes en el contexto académico. El éxito del estudiante no depende solamente de la capacidad intelectual que puede desarrollar; sino en gran medida, está influenciado por la capacidad emocional. Es fundamental para los estudiantes alcanzar un equilibrio entre la inteligencia intelectual y la IE (Altwijri et ál., 2021). La IE adquiere mucha importancia en el rendimiento académico (RA) de los estudiantes universitarios, y se refleja en el promedio ponderado obtenido según los reportes de estudios realizados en varios contextos peruanos, que informan de un RA regular (11-15 puntos) $(69 \%, 67,5 \%$ y $39,5 \%)$ (Castro, 2018 ; Palomino et ál., 2021; Chilca, 2017). 
En la Universidad Nacional Autónoma de Chota (UNACH), una proporción considerable de estudiantes presentaban dificultades para enfrentarse a situaciones nuevas, desinterés, frustración, baja autoestima, deficiente capacidad de resiliencia, dificultad para trabajar en equipo, inadecuadas relaciones interpersonales, conductas disruptivas y bajo RA; lo que generó el interés por el desarrollo de la investigación, que servirá de insumo para sugerir estrategias contextualizadas para la gestión eficaz de las emociones en los universitarios y en consecuencia la mejora de su RA.

La pregunta de investigación fue: ¿Cuál es la relación entre inteligencia emocional y rendimiento académico en los estudiantes de la Universidad Nacional Autónoma de Chota? y la hipótesis planteada: La relación entre inteligencia emocional y rendimiento académico en los estudiantes de la Universidad Nacional Autónoma de Chota, es significativa.

El objetivo fue relacionar la inteligencia emocional y el rendimiento académico en los estudiantes de la Universidad Nacional Autónoma de Chota, Perú.

\section{Método}

\subsection{Enfoque}

El estudio se desarrolló cumpliendo los lineamientos del enfoque cuantitativo, lo que permitió responder al objetivo y a la hipótesis planteada haciendo uso del análisis estadístico de datos.

\subsection{Participantes}

La población fue de 1467 estudiantes matriculados en las escuelas profesionales de Enfermería, Contabilidad, Ingeniería Agroindustrial, Ingeniería Civil e Ingeniería Forestal y Ambiental de la UNACH. Se realizó el cálculo del tamaño muestral para una proporción y se obtuvo una muestra de 325 estudiantes de I ciclo a VIII ciclo [con una proporción esperada del $50 \%$, nivel de confianza del $95,8 \%$ y una precisión del 5\%], que fueron seleccionados mediante un muestreo aleatorio estratificado. Se excluyeron a los estudiantes de IX y $\mathrm{X}$ ciclo por estar realizando prácticas preprofesionales exclusivas en entidades externas a la universidad.

\subsection{Tipo de investigación}

Estudio descriptivo correlacional, el propósito fue establecer la relación entre las dos variables investigadas, IE y RA, además no hubo intervención por parte de los investigadores que modifiquen el comportamiento de las variables.

\subsection{Diseño de investigación}

El diseño de investigación fue no experimental transversal, dado que los datos de ambas variables se recolectaron en una sola oportunidad, por única vez.

\subsection{Técnicas}

Las técnicas de recolección de datos fueron la psicometría para la IE y el análisis documental para el RA. La psicometría fue autoadministrada (los participantes por sí mismos desarrollaban los ítems), sincrónica (el desarrollo de los ítems fue en el momento solicitado por los investigadores) y confidencial (se mantuvo en reserva la información de identificación de los participantes). El análisis documental se aplicó haciendo uso de una ficha de recolección de datos.

\subsection{Instrumentos}

El instrumento utilizado para medir la IE fue el Test de Inteligencia Emocional de BarOn ICE, adaptado a Perú por Ugarriza y Pajares (2005), consta de 60 ítems divididos en 5 dimensiones: Intrapersonal 
(ítem 1 al ítem 12), interpersonal (ítem 13 al ítem 24), adaptabilidad (ítem 25 al ítem 36), manejo del estrés (ítem 37 al ítem 48) y estado de ánimo (ítem 49 al ítem 60). Cada ítem presenta valores finales que van de 1 a 4 puntos: 1 equivale a Muy rara vez, 2 a Rara vez, 3 a A menudo y 4 a Muy a menudo. Los ítems inversos son: ítem 5, ítem 38, ítem 40, ítem 41, ítem 42, ítem 43, ítem 44, ítem 45, ítem 46, ítem 48, ítem 56. El puntaje global se clasifica: de 60 a 156 IE baja, de 157 a 178 IE promedio y de 179 a 240 IE alta. Se utilizó este instrumento porque la cantidad de reactivos fue la más adecuada para su aplicación, así mismo incluye a adolescentes hasta sus primeros años de maduración (en la investigación la edad mínima fue de 16 años con una media de 20 años), es la etapa en la que se incorporan a la vida universitaria, y previo a su aplicación se realizó una prueba piloto para garantizar la validez de respuesta.

Para adaptar el instrumento al contexto, se realizó una prueba piloto en 33 estudiantes de la Universidad Nacional de Cajamarca Sede Chota, quienes tenían las mismas características de las unidades de estudio. La fiabilidad del instrumento mediante la prueba Alfa de Cronbach fue de 0,837 , óptimo para ser aplicado en la muestra.

Para medir el RA se utilizó una ficha de recolección de datos de elaboración propia, que consignaba: nombres y apellidos, carrera profesional, ciclo académico, código de estudiante y promedio ponderado. Se consideró los promedios ponderados de cada estudiante, obtenidos durante el semestre académico 2017 - I (Oficina de Registros Académicos de la UNACH, 2017), mediante escala vigesimal con nota aprobatoria de 11 .

\subsection{Procedimientos}

El estudio se desarrolló durante los meses de agosto a octubre del año 2017. Para el recojo de los datos se solicitó el permiso correspondiente a las autoridades de la UNACH, luego a los docentes para ingresar a las aulas y aplicar el Test de IE; los estudiantes seleccionados recibieron una carta de invitación y quienes deseaban participar voluntariamente, firmaron el consentimiento informado.

La información del RA fue proporcionada por el responsable de la Oficina de Registros Académicos de la UNACH (2017), previa autorización.

\subsection{Análisis estadístico}

Los datos recolectados fueron ingresados en una matriz creada en el software estadístico SPSS v. 24 para Windows. Para el análisis univariado se determinaron frecuencias absolutas y relativas, medidas de tendencia central y de dispersión. Para el análisis bivariado se utilizó la prueba estadística Rho de Spearman y se consideró estadísticamente significativo un $\mathrm{p}$-valor $<0,05$.

\subsection{Aspectos éticos}

Los estudiantes debidamente informados y de forma voluntaria firmaron el consentimiento informado para participar en la investigación. Los datos se manejaron discretamente codificando su identidad, manteniendo la confidencialidad y reserva de la información en todo momento.

\section{Resultados}

Se trabajó con 325 estudiantes de ambos sexos, pertenecientes a los ciclos académicos de I ciclo a VIII ciclo de la UNACH y con una edad promedio de 20,3 años $\pm 2,2$. A partir de los 26 años la frecuencia fue de uno por año, los que constituían valores atípicos, dado que el $95 \%$ presentó una edad alrededor de los 20 años (tabla 1). 
Tabla 1

Edad de los estudiantes de la Universidad Nacional Autónoma de Chota

\begin{tabular}{|c|c|c|c|c|}
\hline Edad & No $^{\circ}$ & $\begin{array}{c}\text { Mín. - } \\
\text { Máx. }\end{array}$ & Media \pm DS & IC 95\% \\
\hline Años & 325 & $16-35$ & $20,3 \pm 2,2$ & $20,1-20,5$ \\
\hline
\end{tabular}

Los estudiantes universitarios presentaron porción superior a la mitad, seguido de IE una IE alta $(51,1 \%)$, con una ligera pro- promedio $(40,6 \%)$ (tabla 2$)$.

Tabla 2.

Inteligencia emocional de los estudiantes de la Universidad Nacional Autónoma de Chota

\begin{tabular}{|c|c|c|}
\hline Inteligencia emocional & $\mathbf{N}^{\circ}$ & $\%$ \\
\hline Baja & 27 & 8,3 \\
\hline Promedio Total & 132 & 40,6 \\
\hline Alta & 166 & 51,1 \\
\hline \multicolumn{2}{|c|}{$\mathbf{3 2 5}$} & $\mathbf{1 0 0 , 0}$ \\
\hline
\end{tabular}

Fuente: Test de inteligencia emocional aplicado a estudiantes universitarios

En relación al RA los estudiantes univer- cuartil inferior, en una escala de calificasitarios presentaron un promedio ponde- ción vigesimal (tabla 3).

rado de $12,59 \pm 1,17$, nota aprobatoria del

Tabla 3.

Rendimiento académico de los estudiantes de la Universidad Nacional Autónoma de Chota

\begin{tabular}{ccccc}
\hline Rendimiento académico & No & Mín. - Máx. & Media \pm DS & IC $95 \%$ \\
\hline Promedio ponderado & 325 & $8-16$ & $12,59 \pm 1,17$ & $12,46-12,72$ \\
\hline
\end{tabular}

Fuente: Actas de notas de estudiantes universitarios

La relación entre IE y RA en los estudian- solo se obtuvo una relación estadística tes de la UNACH fue significativa ( $p=$ significativa entre la adaptabilidad y el RA $0,043)$, a nivel de dimensiones de la IE, $\quad(p=0,021)$ (tabla 4$)$. 


\section{Tabla 4}

\section{Relación entre inteligencia emocional y rendimiento académico en los estudiantes de la Universidad Nacional Autónoma de Chota}

\begin{tabular}{|l|c|c|c|}
\hline \multicolumn{1}{|c|}{ Variables } & Prueba estadística & $\begin{array}{c}\text { Coeficiente de } \\
\text { correlación }\end{array}$ & p-valor \\
\hline IE (global) y RA & Rho de Spearman & 0,112 & $0,043^{*}$ \\
\hline Intrapersonal y RA & Rho de Spearman & 0,091 & 0,100 \\
\hline Interpersonal y RA & Rho de Spearman & 0,073 & 0,188 \\
\hline Adaptabilidad y RA & Rho de Spearman & 0,128 & $0,021^{*}$ \\
\hline Manejo del estrés y RA & Rho de Spearman & 0,055 & 0,320 \\
\hline Estado de ánimo y RA & Rho de Spearman & 0,032 & 0,562 \\
\hline
\end{tabular}

IE: inteligencia emocional, RA: rendimiento académico.

${ }^{*} p<0,05$.

\section{Discusión}

Los resultados de la IE no distan de manera importante con los encontrados por PaIomino (2018), con el $86,8 \%$ de IE regular en los estudiantes. Asimismo, los estudios de Loyola (2018) y Nuñez (2018), también reportan resultados de IE promedio en los estudiantes, con el $72 \%$ y $37,9 \%$ respectivamente, por tratarse de estudios realizados dentro del contexto nacional, los cuales comparten las mismas características del sistema educativo.

Una adecuada IE en los estudiantes mejora las relaciones interpersonales, favorece la adaptación, la capacidad de resiliencia, el trabajo en equipo, las habilidades de comunicación y las habilidades de aprendizaje para tener un mejor RA (Barragán et ál., 2021). Los estudiantes con una IE óptima reconocen las emociones de sí mismos y de las personas de su entorno, saben gestionarlas de manera que éstas no les afecten, manifiestan sus emociones y sentimientos de manera asertiva y son capaces de controlarlas tanto en sus actividades cognitivas como sociales facilitando la ejecución de las mismas. Por ello, el éxito de los estudiantes en las relaciones interpersonales y en distintas áreas de la vida, depende de la estructura de su competencia emocional (Yu et ál., 2021).
Los estudiantes en el ámbito universitario experimentan emociones positivas como interés, entusiasmo, alegría y/o satisfacción (que va a permitir al estudiante desarrollar tareas, solucionar problemas y favorecer la autorregulación) y negativas como impotencia, inseguridad, aburrimiento, miedo y decepción (que van a interferir en su RA y en la toma de decisiones) (Pulido y Herrera, 2017). Las emociones mal gestionadas alteran la conducta y el aprendizaje en los estudiantes, que podrían conllevar al fracaso y deserción universitaria. Por ello, es necesario que los tutores y docentes fortalezcan las habilidades emocionales de los estudiantes de manera continua y progresiva.

Respecto al RA, el promedio ponderado fue aprobatorio, encontrándose en el cuartil inferior; resultados que guardan relación con los estudios de Palomino et ál. (2021), Castro (2018), Núñez (2018), Palomino (2018), Loyola (2018) y Chilca (2017), quienes reportan promedios ponderados parecidos, debido a que la aplicación y ejecución de políticas educativas son verticales en el país y no consideran los factores subyacentes que pueden estar influyendo en cada contexto particular.

El RA de los estudiantes puede estar influenciado por diferentes factores, adicio- 
nalmente al manejo ineficaz de las emociones, como la desmotivación en las asignaturas, métodos de estudio inadecuados, dificultades de atención y comprensión, material didáctico poco claro, trabajar y estudiar al mismo tiempo e incluso la vocación profesional errónea, conllevarían a un deficiente RA (Chisholm-Burns et ál., 2021; Larabi-Marie-Sainte et ál., 2021). Según Carrillo et ál. (2017), los avances tecnológicos puestos a disposición de los estudiantes como el uso excesivo del internet y el teléfono móvil, constituyen los principales distractores que interrumpen sus actividades académicas y sus deberes como estudiantes.

El sistema de calificación universitaria en el Perú en la mayoría de universidades es el sistema vigesimal de 0 a 20 , con nota mínima aprobatoria de 11. No obstante, universidades como la Universidad Católica Los Ángeles de Chimbote (2018) y la Universidad Científica del Sur (2017), consideran la nota mínima aprobatoria de 13. En este sentido, el RA obtenido en los estudiantes apenas bordea la nota mínima aprobatoria, por lo que se debe fortalecer las relaciones interpersonales entre estudiantes y docentes con la finalidad de reorientar el trabajo colaborativo y el aprendizaje eficaz.

El estudio encontró una relación estadísticamente significativa entre la IE y el RA en los estudiantes universitarios participantes. Resultados similares a los encontrados por Carrillo-Galán (2020), Loyola (2018), Nuñez (2018), Camones (2018) y Palomino (2018), con p-valor altamente significativos, indicativo de que estas dos variables se relacionan independientemente del contexto académico estudiado. No obstante, en este estudio a diferencia de los otros la correlación entre variables fue débil, debido probablemente a que la IE alta ligeramente sobrepasa la mitad de participantes, y el promedio ponderado prevalece en el cuartil inferior aprobatorio.
Por otro lado, respecto a la relación entre las dimensiones de la IE y el RA solamente fue significativa la relación entre adaptabilidad y el RA con una correlación débil, puesto que los puntajes en las dimensiones de la IE se concentraron en el nivel medio y a diferencia de otros estudios, Palomino (2018) reporta una relación significativa entre todos los componentes de la IE y el RA, los demás solo reportan la relación de la IE global con el RA.

La adaptabilidad de los estudiantes universitarios se produce paulatinamente, según se incorporan al sistema educativo superior, sin embargo, este proceso no ocurre con el mismo ritmo en todos los estudiantes, por lo que puede ser diferenciado según las características individuales, familiares y sociales de cada cual. En ese sentido, es necesario que las autoridades universitarias brinden los mecanismos pertinentes para que exista una inducción oportuna y eficiente en esta nueva etapa de los estudiantes y logren así el RA deseado (Álvarez-Pérez y López-Aguilar, 2020).

La educación racional enfocada solo en el estudio de contenidos, aislada del campo de las emociones, no contribuirá en la formación integral del futuro profesional, porque las relaciones humanas son el principal aliciente para el buen desempeño y bienestar personal y social del estudiante. Los estudiantes que gestionan eficazmente sus emociones y sentimientos, saben comprender y tienen la capacidad de relacionarse con los sentimientos de los demás de forma empática, suelen sentirse más satisfechos y capaces de dominar los hábitos mentales que determinan el RA (Niño et ál., 2017).

Los estudiantes emocionalmente inteligentes manifiestan amor propio, orgullo, tienen una valoración positiva de sí mismos, se sientes satisfechos e interactúan con su entorno de manera satisfactoria, tienden a apoyar a sus compañeros y 
tienen menos posibilidades de expresar comportamientos y actitudes negativas que pueden conllevar a la violencia y a la frustración. Afrontar de forma adecuada situaciones de estrés generadas en el ambiente académico va a influir positivamente en el RA (Cheema et ál., 2021).

Por consiguiente, se puede inferir que los estudiantes con puntuaciones altas en IE tienden a obtener mejores resultados en su RA. Es decir, al tener una mayor capacidad de reconocimiento, entendimiento y regulación de las emociones, los estudiantes serán capaces de creer en su propia capacidad para hacer frente a los aspectos negativos y tener un mejor desempeño académico.

\section{Conclusiones}

Se concluye que la IE de la mayor proporción de estudiantes universitarios de la UNACH fue de nivel alto, indicador de que manejan adecuadamente sus emociones, no obstante, debe fortalecerse el desarrollo de estrategias en el manejo de la IE para tener un alcance totalitario. EI RA promedio de los estudiantes fue de 12,59 de puntaje ponderado. Finalmente, la IE y la dimensión adaptabilidad se relacionaron significativamente con el RA de los estudiantes, aportándose evidencia a favor de la hipótesis planteada.

EI RA de los estudiantes levemente sobrepasa la nota aprobatoria mínima; por lo tanto, se hace necesaria la investigación de factores que pueden estar influyendo en esta variable, como el contexto familiar, amical y personal, con la finalidad de aprovechar su IE favorable en la gestión adecuada de sus actividades académicas que permita mejorar su RA.

El estudio se desarrolló en una sola universidad y con una muestra, constituyéndose en las limitaciones, que fueron suplidas mediante el muestreo aleatorio estra- tificado, garantizando representatividad. Así mismo como alcance del estudio, los resultados confirman lo reportado en otras investigaciones y en otros contextos; no obstante, es necesario buscar la asociación del RA con otras variables que pueden estar injiriendo de manera importante y conjunta en su manifestación, pues por ser un fenómeno multifactorial su asociación con la IE tan solo constituye el punto de partida para un trabajo integral, coordinado y sostenible de una educación de calidad en los estudiantes.

\section{Referencias bibliográficas}

Ain, N. U., Munir, M., Suneel, I. (2021). Role of emotional intelligence and grit in life satisfaction. Heliyon, 7(4), e06829. https://doi.org/10.1016/j.heliyon.2021. e06829

Altwijri, S., Alotaibi, A., Alsaeed, M., Alsalim, A., Alatiq, A., Al-Sarheed, S., Agha, S., Omair, A. (2021). Emotional Intelligence and its Association with Academic Success and Performance in Medical Students. Saudi J Med Med Sci, 9(1), 31-37. https://doi.org/10.4103/sjmms.sjmms_375_19

Álvarez-Pérez, P. R., López-Aguilar, D. (2020). Competencias de adaptabilidad y factores de éxito académico del alumnado universitario. Rev. iberoam. educ. super, 11(32), 46-66.

https://doi.org/10.22201/ii-

sue.20072872e.2020.32.815

Barragán, A. B., Pérez-Fuentes, M. C., Molero, M. M., Martos, Á., Simón, M. M., Sisto, M., Gázquez, J. J. (2021). Emotional Intelligence and Academic Engagement in Adolescents: The Mediating Role of Self-Esteem. Psychol Res Behav Manag, 14, 307-316.

https://doi.org/10.2147/PRBM.S302697 
Camones, F. C. (2018). Efecto de la inteligencia emocional en el rendimiento académico en estudiantes universitarios [tesis de maestría, Universidad César Vallejo]. Repositorio Institucional UP.

https://alicia.concytec.gob.pe/vufind/Record/UCVV_adf02136ad7f18abb44a5aadb8aeacb8

Carrillo-Galán, Y. M., Pérez-Vega, J., Roa-González, S. C., Morales-González, Y., Rylander-Yamada, J., Méndez-Quevedo, T. J. (2020). Inteligencia emocional y rendimiento académico en estudiantes universitarios. Rev Mex Med Forense, 5(Suppl:3), 161-164.

https://www.medigraphic.com/cgi-bin/new/ resumen.cgi?IDARTICULO=97761

Carrillo, M. G., Zúñiga, B. L., Toscano, B. A., Aguas, N., Díaz, J. C. (2017). Los dispositivos móviles e Internet y su uso en el aula: ¿Un factor distractor en el proceso de aprendizaje? Un estudio de caso. Tecnología Educativa Revista CONAIC, IV(3), 40-51.

https://www.conaic.net/revista/publicaciones/Vol_IV_Num3_2017/Articulo4.pdf

Castro, E. M. (2018). Rendimiento académico y estrés en estudiantes de enfermería, Universidad Nacional Toribio Rodríguez de Mendoza, Chachapoyas - 2017 [tesis de licenciatura, Universidad Nacional Toribio Rodríguez de Mendoza]. Repositorio Institucional UN. http://repositorio. untrm.edu.pe/handle/UNTRM/1397

Cheema, S., Maisonneuve, P., Abraham, A., Chaabna, K., Tom, A., Ibrahim, H., Mushannen, T., Yousuf, W., Lowenfels, A. B., Mamtani, R. (2021). Factors associated with perceived stress in Middle Eastern university students. J Am Coll Health, 1-8. https://doi.org/10.1080/07448481.2020.18 65979

Chilca, M. L. (2017). Autoestima, hábitos de estudio y rendimiento académico en estudiantes universitarios. Propósitos y representaciones, 5(1), 71-127.

http://dx.doi.org/10.20511/pyr2017. v5n1.145

Chisholm-Burns, M. A., Berg-Poppe, P., Spivey, C. A., Karges-Brown, J., Pithan, A. (2021). Systematic review of noncognitive factors influence on health professions students' academic performance. Adv Health Sci Educ Theory Pract.

https://doi.org/10.1007/s10459-02110042-1

Larabi-Marie-Sainte, S., Jan, R., Al-Matouq, A., Alabduhadi, S. (2021). The impact of timetable on student's absences and performance. PLoS One, 16(6), e0253256.

https://doi.org/10.1371/journal. pone.0253256

Loyola, N. L. (2018). Inteligencia emocional y el rendimiento académico en los alumnos del VIII ciclo de la Facultad de Enfermería de la Universidad Nacional Federico Villarreal - Lima, 2017 [tesis de maestría, Universidad Nacional de Educación Enrique Guzmán y Valle]. Repositorio Institucional UN. https://repositorio.une. edu.pe/handle/UNE/2187

Niño, J. I., García, E., Caldevilla, D. (2017). Inteligencia emocional y educación universitaria: Una aproximación. Revista de Comunicación de la SEECI, (43), 15-27.

https://dialnet.unirioja.es/descarga/articulo/6073590.pdf

Nuñez, P. P. (2018). Estudio relacional entre inteligencia emocional y rendimiento académico de estudiantes de I A VIII semestre de Psicología Humana en una Universidad Privada de Arequipa, 2017 [tesis doctoral, Universidad Nacional de San Agustín]. Repositorio Institucional UN. http://repositorio.unsa.edu.pe/handle/ UNSA/7238 
Ocoruro, D. N. (2021). Inteligencia emocional y estrés académico en estudiantes de la Escuela Profesional de Psicología de la Universidad Alas Peruanas Juliaca - 2019 [tesis de licenciatura, Universidad Autónoma de Ica]. Repositorio Institucional UN.

http://repositorio.autonomadeica.edu. pe/bitstream/autonomadeica/885/1/Dalila\%20Noemi\%20Ocoruro\%20Quico.pdf

Oficina de Registros Académicos de la Universidad Nacional Autónoma de Chota. (2017). Promedios ponderados de estudiantes en el semestre académico 2017 - I. Oficina de Registros Académicos de la Universidad Nacional Autónoma de Chota.

Palomino, G. S. (2018). Inteligencia emocional y rendimiento académico, en los estudiantes de la escuela profesional de estadística de la Universidad Nacional Pedro Ruiz Gallo. 2017 - I [tesis de maestría, Universidad Nacional Pedro Ruiz Gallo]. Repositorio Institucional UN.

https://repositorio.unprg.edu.pe/bitstream/handle/20.500.12893/7650/BCTES-3426\%20PALOMINO\%20ARCE.pdf?sequence $=1$ \&isAllowed $=y$

Palomino, J. A., Zevallos, G., Orizano, L. A. (2021). Estilos de vida saludable y rendimiento académico en estudiantes universitarios. Delectus, 4(1), 82-92.

https://doi.org/10.36996/delectus.v4i1.103

Pulido, F. \& Herrera, F. (2017). La influencia de las emociones sobre el rendimiento académico. Ciencias Psicológicas, 11(1), 29-39.

https://revistas.ucu.edu.uy/index.php/cienciaspsicologicas/article/view/1344/1334
Tariq, S., Tariq, S., Atta, K., Rehman, R., Ali, Z. (2020). Emotional Intelligence: A predictor of undergraduate student's academic achievement in altered living conditions. J Pak Med Assoc, 70(12(B)), 23982402. https://doi.org/10.47391/JPMA.429

Ugarriza, N., Pajares, L. (2005). La evaluación de la inteligencia emocional a través del inventario de BarOn ICE: NA, en una muestra de niños y adolescentes. Persona, (8), 11-58. https://www.redalyc. org/pdf/1471/147112816001.pdf

Universidad Católica Los Ángeles Chimbote. (2018). Reglamento académico. Universidad Católica Los Ángeles Chimbote. https://www.uladech.edu.pe/index.php/ transparencia/category/82-reglamento-de-la-universidad.html

Universidad Científica del Sur. (2017). Reglamento de estudios de pregrado. Universidad Científica del Sur.

https://www.cientifica.edu.pe/sites/default/ files/reglamento_de_estudios_pregrado_1.pdf

Yu, M. L., Brown, T., Hewitt, A., Cousland, R., Licciardi, L., Lyons, C. (2021). Baccalaureate occupational therapy students' development of social and emotional competencies. Nurse Educ Today, 105, 105032. https://doi.org/10.1016/j. nedt.2021.105032 